\title{
SPHERICAL-RADIAL MULTIPLIERS ON THE HEISENBERG GROUP
}

\author{
M.E. EGWE* \\ Department of Mathematics, University of Ibadan, Ibadan, Nigeria \\ *Corresponding author: murphy.egwe@ui.edu.ng

\begin{abstract}
Let $\mathbb{H}_{n}$ be the $(2 n+1)$-dimensional Heisenberg group. We consider a radial Fourier multiplier which is a spherical function on $\mathbb{H}_{n}$ and show that it is a Herz-Schur multiplier.
\end{abstract}

\section{INTRODUCTION}

The Theory of multipliers has grown over the years to yield several results and applications in virtually all aspects of Analysis and Mathematics in general. Its use in Harmonic Analysis has assumed an enormous dimension. The theory was introduced on the Heisenberg group by G. Mauceri [13] and several other authors. Recently, Bagchi [2] revisited Fourier multipiers on the Hesisenberg group showing some variance of the results of [14] and [15]. A transference result of Fourier multipliers from $S U(2)$ to the Heisenberg group was considered by F.Ricci [15].

The spherical functions form a large subject matter on this group [3], [1]. A construction of spherical radial functions on the Heisenberg group was given in [5], [6] and [7].

The concept of Schur multipliers or completely bounded functions has attained an exciting peak in Harmonic Analysis. However, the version of the result we shall consider in this work can be seen in [4].

Received March 25 ${ }^{\text {th }}, 2020$; accepted April 16 ${ }^{\text {th }}, 2020$; published June 17 $7^{\text {th }}, 2020$.

2010 Mathematics Subject Classification. 43A90, 42A45, 22E46.

Key words and phrases. spherical-radial multipliers; Herz-Schur; Heisenberg group.

(C)2020 Authors retain the copyrights of their papers, and all open access articles are distributed under the terms of the Creative Commons Attribution License. 


\section{MAIN RESUlT}

Definition 2.0.1 [2]: Given a bounded measurable function $m(\eta)$ on $\mathbb{R}^{n}$, we can define a transformation $T_{m}$ by setting

$$
\left(\widehat{T_{m} f}\right)=m(\eta) \hat{f}(\eta), \quad f \in L^{2}\left(\mathbb{R}^{n}\right) .
$$

By Placherel Theorem, $T_{m}$ is a bounded operator on $L^{2}\left(\mathbb{R}^{n}\right)$.

Definition 2.0.1: Let $p \in[0, \infty)$, if $m$ is a continuous function on $\mathbb{R}^{n}$ such that $\forall \epsilon>0$, the operators

$$
\left(\widehat{M_{\epsilon}} f\right)=m\left(\epsilon^{-1} n\right) \hat{f}(n)
$$

are uniformly bounded multiplier operator on $L^{p}\left(\mathbb{T}^{n}\right)$, then $m$ defines a bounded multiplier operator on $L^{p}\left(\mathbb{R}^{n}\right)$.

When $T_{m}$ extends to $L^{p}\left(\mathbb{R}^{n}\right)$ as a bounded operator, we say that $m$ (or equivalently $T_{m}$ ) is a Fourier multiplier for $L^{p}\left(\mathbb{R}^{n}\right)$.

Theorem 2.0.3 (Hormander's Multiplier Theorem): Let $k=\left[\frac{n}{2}\right]+1$ and $m$ be of class $C^{k}$ away from the origin. If for any $\beta \in N^{n}$ satisfying $|\beta|<k$, we have

$$
\sup _{R} R^{|\beta|-\frac{n}{2}}\left(\int_{\mathbb{R}^{n}}\left|D^{\beta} m(\eta)\right|^{2} \chi_{\{R<|\eta|<2 R\}}(\eta) d \eta\right)^{1 / 2}<\infty
$$

then $m$ is a Fourier multiplier for $L^{p}\left(\mathbb{R}^{n}\right)$ for $1<p<\infty$. In particular, if $\left|D^{\beta} m(\eta)\right| \leq c|\eta|^{-|\beta|}$, then $m$ is an $L^{p}$-multiplier, $1<p<\infty$.

\subsection{The Heisenberg Group $\left(\mathbb{H}_{n}\right)$}

Define the Heisenberg group of dimension $(2 n+1)$ by $\mathbb{H}_{n}=\mathbb{C} \times \mathbb{R}$ equipped with the group law

$$
(z, t)\left(z^{\prime}, t^{\prime}\right)=\left(z+z^{\prime}, t+t^{\prime}+\frac{1}{2} \Im z \cdot z^{\prime}\right), z \cdot z^{\prime}=\sum_{j=1}^{n} z_{j} \cdot \bar{z}_{j}^{\prime} \quad t \in \mathbb{R}, z \in \mathbb{C} .
$$

This gives a two-step nilpotent Lie group with centre given by

$$
\mathfrak{Z}=\{(0, t): t \in \mathbb{R}\}
$$

Full details on the ubiquity of this group can be found in [12] [9], [17], [14].

For each $\mu>0$, we have two non-equivalent irreducible representations of $\mathbb{H}_{n}$ on the Fock space $\mathfrak{F}^{\mu}$ consisting of the entire functions $F$ on $\mathbb{C}^{n}$ such that

$$
\|F\|_{\mathfrak{F}^{\mu}}^{2}=\frac{\mu}{\pi} \int_{\mathbb{C}}|F(w)|^{2} \mathrm{e}^{-\mu|w|^{2}} d w<\infty
$$


These representations have the form [15]

$$
\left.\begin{array}{c}
\left(\rho^{\mu}(\gamma, t) F\right)(w)=\mathrm{e}^{\mu\left(i t+\gamma w+\frac{1}{2}|\gamma|^{2}\right)} F(w+\gamma) \\
\left(\rho^{\mu}(\gamma, t) F\right)(w)=\mathrm{e}^{\mu\left(-i t-\gamma w+\frac{1}{2}|\gamma|^{2}\right)} F(w-\bar{\gamma}) .
\end{array}\right\}
$$

The monomials $\eta_{j}^{\lambda}(w)=\left(\frac{\mu^{j}}{j !}\right)^{1 / 2} w^{j}$ form a orthonormal basis for $\mathfrak{F}^{\mu}$ and the matrix entries corresponding to the representations with respect to the monomials is given by

$$
\tau_{i j}^{ \pm \mu}(\mu, t)=\left\langle\rho^{ \pm \mu}(\gamma, t) \eta_{i}^{\mu}, \eta_{j}^{\mu}\right\rangle
$$

Now, let $d u=\left(\frac{1}{2 \pi^{2}}\right) d z d t$ denote the normalized Haar measure on $\mathbb{H}_{n}$. Then, given an integrable function $f$ on $\mathbb{H}_{n}$ and a nonzero real number $\mu$, we have a countably infinite matrix with $(i, j)$ entry given by

$$
\hat{f}(\mu, i, j)=\int_{\mathbb{I H}_{n}} f(u) \overline{\tau_{i j}^{\mu}}(u) d u
$$

With this normalisation and matrix entries, we obtain the Plancherel formula given by

$$
\int_{\mathbb{I H}_{n}}|f(u)|^{2} d u=\int_{\infty}^{\infty} \sum_{i, j=0}^{\infty}|\hat{f}(\mu, i, j)|^{2}|\mu| d \mu .
$$

We now give the following definition following Hormander's theorem.

Definition 2.1.1: Let $\mu \neq 0$ and $m(\mu)$ a countably infinite matrix with entries $m(\mu, i, j)$ that are measurable in $\mu$ for each $i, j$. We say that this induces a bounded multiplier on $L^{p}\left(H_{n}\right)$ if

$$
\|M f\|_{p} \leq c\|f\|
$$

where $\widehat{(M f)}(\mu)=\hat{f}(\mu) m(\mu)$ for some $f$ in some dense subspace of $L^{p}\left(\mathbb{H}_{n}\right)$.

In what follows, we shall construct the spherical radial multipliers following [5], [6].

Let $\varphi_{\lambda}^{K}$ be a $K$-spherical function on $\mathbb{H}_{n}$. That is the distinguished spherical function restricted to $L^{1}(K \backslash G / K)$ where $(K, G)$ is a Gelfand pair, $K$ a compact subgroup of $A u t\left(\mathbb{H}_{n}\right)$. In this case, $G$ may be taken as a semi-direct product of $K$ and $\mathbb{H}_{n}$ denoted as $G:=K \rtimes \mathbb{H}_{n}$ [1].

Now, recall that the Heisenberg group heat equation defined on $\mathbb{H}_{n} \times \mathbb{R}^{+}$is given by

$$
\partial_{t} U(u, t)=\triangle U(u, t), U(u, t) \in \mathbb{H}_{n} \times \mathbb{R}^{+} .
$$

The fundamental solution of (12) is given in [16] as

$$
K_{t}(x, u, \xi)=c_{n} \int_{\mathbb{R}} \mathrm{e}^{\lambda E} \mathrm{e}^{-t \lambda^{2}}\left(\frac{\lambda}{\sinh \lambda t}\right) \mathrm{e}^{\frac{1}{4} \lambda(\operatorname{coth} t \lambda)(x . x+u . u)} d \lambda
$$


where $c_{n}=(4 \pi)^{-n}, \lambda \in \mathbb{R}^{*}:=\mathbb{R} \backslash\{0\}$. By a unique transformation of $K_{t}(x, u, \xi)$ given explicitly in [6], we obtain that

$$
K_{t}(u)=c_{n} t^{-n / 2} \varphi_{\lambda}^{K}(u) \delta_{r}^{-2}(u) \mathrm{e}^{\frac{|u|^{2}}{4 t}}
$$

This gives a representations in (8) of $\mathbb{H}_{n}$ with respect to the dilations on the group.

Thus, (9) becomes

$$
\hat{f}(\mu, i, j)=\int_{\mathbb{H}_{n}} f(u) \overline{K_{i j}^{\lambda}}(u) d u
$$

where (from (14) we have)

$$
K_{i j}^{\lambda}=\left\langle\varphi_{\lambda}^{K}(\xi, t) \eta_{i}^{\lambda}, \eta_{j}^{\lambda}\right\rangle
$$

The spherical transforms of a function on $\mathbb{H}_{n}$ are then obtained and given as [1], [5]:

$$
\tilde{f}(\lambda, t)=\int_{\mathbb{H}_{n}} f(z, t) \overline{\varphi_{\lambda}^{K}}(z, t) d z d t
$$

and

$$
\tilde{f}(0, \rho)=\int_{\mathbb{H}_{n}} f(z, t) \mathcal{J}_{0}^{\rho}(z) d z d t
$$

where

$$
\varphi_{\lambda}^{K}=\mathrm{e}^{2 \pi i \lambda t} \mathrm{e}^{-2 \pi|\lambda||z|^{2}} \prod_{j=1}^{n} \mathcal{L}_{k}^{0}\left(4 \pi|\lambda|\left|z_{j}\right|^{2}\right), \lambda \in \mathbb{R}^{*}, k \in\left(\mathbb{Z}_{+}\right)^{n}
$$

and

$$
\mathcal{J}_{0}^{\rho}=\prod_{j=1}^{n} J_{0}\left(\rho_{j} \cdot\left|z_{j}\right|\right), \rho \in\left(\mathbb{R}_{+}\right)^{n} .
$$

Here, $\mathcal{L}_{k}^{0}$ is the Laguerre polynomial of degree $k$ and $J_{0}$ is the Bessel function (of first kind) of index 0 .

Definition 2.1.2: Let $\mathscr{M}=\left\{M(\lambda) \in \mathscr{B}\left(L^{2}\left(\mathbb{R}^{n}\right)\right): \lambda \in \mathbb{R}^{*}\right\}$ be a family of operators. Suppose that $T_{M}$ is the corresponding group Fourier multiplier. Also, let $\varphi_{M}^{K}(\lambda)$ be the spherical Fourier multiplier associated with the parameter and operator $M(\lambda)$. Then, it becomes clear that

$$
T_{M} f(z, t)=\int_{\mathbb{R}} \mathrm{e}^{-i \lambda t} \overline{\varphi_{M(\lambda)}^{K}}(\lambda) f^{\lambda}(t) d \lambda, \text { for all } f \in L^{1} \cap L^{2}\left(\mathbb{H}_{n}\right) .
$$

This implies that

$$
T_{M} f(z, t)=\int_{\mathbb{R}} \overline{K_{j k}^{\lambda}} f(t) \mathrm{e}^{-i \lambda t} d \lambda, f \in \mathscr{S}\left(\mathbb{H}_{n}\right)[1] .
$$

Definition 2.1.3: We shall say a matrix-valued function $M(\mu)=(M(\mu, i, j))_{i, j \in \mathbb{N}}$ is a bounded Fourier multiplier for $\mathbb{H}_{n}$ if $m(., i, j) \in L^{\infty}(\mathbb{R})$ for every $i, j \in \mathbb{N}$, and if

$$
\|M\|_{\infty}=\operatorname{ess} . \sup \|M(\mu)\|_{\mathcal{L}\left(\ell^{2}\right)}<\infty
$$

This definition together with 2.1.1 yield the following theorem as seen in [14].

Theorem 2.1.4: If $M$ is a bounded Fourier multiplier on $\mathbb{H}_{n}$, the requirement that

$$
\widehat{T_{M} f}(\mu)=\hat{f}(\mu) M(\mu)
$$


defines a bounded left invariant operator $T_{M}$ on $L^{2}\left(\mathbb{H}_{n}\right)$, with $\left\|T_{M}\right\|_{\mathcal{L}\left(L^{2}\left(\mathbb{H}_{n}\right)\right)}=\|M\|_{\infty}$. Conversely, for any bounded left-invariant operator $T$ on $L^{2}\left(\mathbb{H}_{n}\right)$, there is a bounded Fourier multiplier $M$ such that $T=T_{M}$.

Definition 2.1.5: A function $f: \mathbb{R}^{n} \longrightarrow \mathbb{R}$ is said to be radial if there is a function $\phi$ defined on $[0, \infty)$ such that $f(x)=\phi(|x|)$ for almost every $x \in \mathbb{R}^{n}$.

Simple and classical examples of radial functions and their properties can be seen in for example [10], [3], [6] and [11].

Thus, given a $K$-spherical function, $\varphi_{\lambda}^{K}$ restricted to $L^{1}(K \backslash G / K)$ where $\left(K, H_{n}\right)$ is a Gelfand pair, $K$ a compact subgroup of $A u t\left(\mathbb{H}_{n}\right)$, then $\varphi_{\lambda}^{K}$ is a unique radial function since it is a radial eigenfunction of $\triangle_{\mathbb{H}_{n}}[6]$, [7]. Thus, $\varphi_{\lambda}^{K}(u)=\psi(|u|)$ This forces forces (15) to become

$$
\begin{aligned}
\varphi_{\lambda}^{K}(u) & =c_{n} \psi\left(\mathrm{e}^{-i \theta}|u|, t\right) \\
& =c_{n} K_{i j}^{\lambda}(|u|, t) .
\end{aligned}
$$

This establishes (21).

In fact, we have the following result [14].

Prposition 2.1.6: $K_{j k}^{\lambda}$ is a unique radial function in $\operatorname{span}\left\{\varphi_{\lambda}^{K}:|\lambda| \in \sum\right\}$, where $\sum$ is the Heisenberg fan, up to scalar multiples.

Definition 2.1.7: Let $\mathbb{H}_{n}$ be the $2 n+1$-dimensional Heisenberg group. Then $f$ on $\mathbb{H}_{n}$ is said to be Herz-Schur, $f \in B_{2}\left(\mathbb{H}_{n}\right)$ if there exist $u, \nu \in \mathbb{H}_{n}$ such that

$$
f\left(u^{-1} \nu\right)=\left\langle\rho_{1}^{\mu}(u), \rho_{2}^{\mu}(\nu)\right\rangle
$$

where $\rho_{1}^{\mu}$ and $\rho_{2}^{\mu}(\nu)$ are irreducible unitary representations of $\mathbb{H}_{n}$ on $L^{2}(\mathbb{R})$. Here, we assume that $\sup _{u \in \mathbb{H}_{n}}\left\|\rho_{1}^{\mu}(u)\right\|<\infty$ and $\sup _{\nu \in \mathbb{H}_{n}}\left\|\rho_{2}^{\mu}(\nu)\right\|<\infty$, where $\|\cdot\|$ is the Fourier multiplier norm equivalent to the Koranyi norm [8].

Theorem 2.1.8 Let $T_{M}$ be the group Fourier multiplier on $\mathbb{H}_{n}$ acting on a $K$-bounded spherical function, $f \in \mathscr{S}\left(\mathbb{H}_{n}\right)$. Then, $T_{M}$ is a Herz-Schur multiplier on $\mathbb{H}_{n}$.

Proof: Following [4], any $f$ can be expressed in the form given in (22). Thus, if we consider (21) above, we readily see that up to scalar multiples, $K_{i j}^{\mu}$ is the unique radial function with $\left\|K_{i j}^{\mu}\right\| \leq 1$. Thus,

$$
\begin{aligned}
\left|T_{M} f(z, t)\right| & =\left|\int_{\mathbb{R}} \overline{K_{i j}^{\mu}} f(t) \mathrm{e}^{-i \mu t} d \mu\right| \\
& \leq \int_{\mathbb{R}}\left|\overline{\left\langle\varphi_{\lambda}^{K}\right.}(\xi, t) \eta_{i}^{\mu}, \eta_{j}^{\mu}\right\rangle f(t) \mathrm{e}^{-i \mu t} d \mu \mid \\
& \leq \sup \left|\overline{\varphi_{\lambda}^{K}(\xi, t)}\right| \int\left|f(t) \mathrm{e}^{-i \mu t} d \mu\right| \\
& =M_{K, \lambda}\|f\|_{\mathscr{S}\left(\mathbb{H}_{n}\right)} .
\end{aligned}
$$


Since the representations of $\mathbb{H}_{n}$ are uniformly bounded on $L^{2}$ and $T_{M}$ is acting on $K$-bounded spherical functions, then the last expression shows that $T_{M} \in B_{2}\left(\mathbb{H}_{n}\right)$ and therefore Herz-Schur.

Conflicts of Interest: The author(s) declare that there are no conflicts of interest regarding the publication of this paper.

\section{REFERENCES}

[1] B. Astengo, D.B. Blasio, and F. Ricci. Gelfand Pairs on the Heisenberg Group and Schwartz Functions. J. Funct. Anal. 256 (5) (2009), 1565-1587.

[2] S. Bagchi. Fourier Multipliers on the Heisenberg groups revisited arXiv:1710.02822v2 [math.CA], 2017.

[3] C. Benson, J. Jenkins. Bounded K-Spherical Functions on the Heisenberg Groups.J. Funct. Anal. 105 (1992), $409-443$.

[4] M. Bozejko, G. Fendler. Herz-Schur multipliers and uniformly bounded representations of discrete groups, Arch. Math. 57 (1991), 290-298.

[5] M.E. Egwe. Aspects of Harmonic Analysis on the Heisenberg group. Ph.D. Thesis, University of Ibadan, Ibadan, Nigeria, 2010.

[6] M.E. Egwe, U.N. Bassey. On Isomorphism Between Certain Group Algebras on the Heisenberg Group, J. Math. Phys. Anal. Geom. 9 (2) (2013), 150-164.

[7] M.E. Egwe. A K-Spherical-Type Solution for Invariant Differential Operators on the Heisenberg Group. Int. J. Math. Anal. 8 (30) 2014, 1475-1486.

[8] M.E. Egwe. The Equivalence of certain norms on the Heisenber group. Adv. Pure Math. 3 (6) (2013), 576-578.

[9] G.B. Folland, Harmonic analysis in phase space, Princeton University Press, Princeton, N.J, 1989.

[10] R. Gangolli. Spherical Functions on Semisimple Lie Groups. In: Symmetric Spaces, W. Boothy and G. Weiss (Eds.). Marcel Dekker, Inc. New York, 1972.

[11] S. Helgason, Groups and geometric analysis: integral geometry, invariant differential operators, and spherical functions, Academic Press, Orlando, 1984.

[12] R. Howe. On the role of the Heisenberg group in harmonic analysis. Bull. Amer. Math. Soc. 3 (1980), 821-843.

[13] G. Mauceri. $L^{p}$-Multipliers on the Heisenberg group. Michigan Math. J. 26 (1979), 361-371.

[14] F. Ricci. Fourier and Spectral Multipliers in $R^{N}$ and in the Heisenberg group. http://homepage.sns.it/fricci/papers/ multipliers.pdf

[15] F. Ricci, R.L. Rubin. Transferring Fourier Multipliers from SU(2) to the Heisenberg Group. Amer. J. Math. 108 (3) (1986), 571-588.

[16] S. Thangavelu. Spherical Means on the Heisenberg Group and a Restriction Theorem for the Symplectic Fourier Transform. Revista Math. Iberoamericana 7 (2) (1991), 135-165.

[17] S. Thangavelu, Harmonic Analysis on the Heisenberg Group, Birkhäuser Boston, Boston, MA, 1998. 